\title{
Congenital complete atrioventricular block from literature to clinical approach - a case series and literature review
}

\author{
Liliana Gozar1, Claudiu Mărginean², Amalia Făgărășan', Iolanda Muntean', Andreea \\ Cerghit-Paler ${ }^{3}$, Dorottya Miklósi ${ }^{3}$, Rodica Togănel ${ }^{1}$
}

${ }^{1}$ Department of Pediatric Cardiology, ${ }^{2}$ Department of Obstetrics and Gynecology, ${ }^{3}$ Pediatric Cardiology, Emergency Institute for Cardiovascular Diseases and Transplantation, "George Emil Palade" University of Medicine, Pharmacy, Science and Technology, Târgu Mureș, Romania

\begin{abstract}
Aim: Congenital atrioventricular block (CAVB) is an immunological condition, secondary to the transfer of maternal Ig $\mathrm{G}$ antibodies from seropositive mothers. Although the presence of these antibodies is high among pregnant women, the prevalence of this fetal pathology is low. The aim of this paper is to analyze a series of cases with intrauterine diagnosis of CAVB and to present their follow-up protocol. Material and method: In the period between 2013-2020, five fetuses were diagnosed and followed up in the Pediatric Cardiology Clinic. In each of the cases, assessment of the hemodynamic status was done by calculation of the fetal cardiovascular profile score (CVPS). In the last cases the follow-up protocol was supplemented with longitudinal speckle tracking evaluation of the ventricular function. Results: In the present series, intrauterine death occurred in one case; in another case resumption of atrioventricular conduction was observed. Epicardial pacemaker implantation was required in three of the patients. Conclusion: Completing the evaluation of ventricular function with the longitudinal speckle tracking method in fetuses and newborn patients with congenital atrioventricular block may play an important role in establishing therapeutic behavior.
\end{abstract}

Keywords: fetal; congenital atrioventricular block; echocardiography

\section{Introduction}

Congenital atrioventricular block can be defined as an impairment of atrioventricular conduction until complete cessation. Thus, ventricular activity is independent of atrial activity, causing a significant decrease in the fetal heart rate, which can lead to intrauterine heart failure and even death. It is known that this fetal pathology is of

Received 17.07.2020 Accepted 17.10.2020

Med Ultrason

2021, Vol. 23, No 2, 188-193

Corresponding author: Claudiu Mărginean

Obstetrics and Gynecology Department,

"George Emil Palade"

University of Medicine,

Pharmacy, Science and Technology

38 Gheorghe Marinescu street,

540139, Târgu Mureș, Romania

Phone: +40-722505311

E-mail: marginean.claudiu@gmail.com autoimmune etiology and is secondary to the transplacental passage of maternal antibodies, especially SSA / Ro and SSB / La [1,2].

Postnatal diagnosis of complete atrioventricular block (CAVB) is made by standard 12 lead electrocardiography, which for technical reasons cannot be performed on the fetus. Thus, echocardiography remains the main diagnostic tool of this intrauterine rhythm disorder. Using the conventional $\mathrm{M}$ mode, obtained by performing a section that includes both the atrial and the ventricular walls, atrio-ventricular activity and dissociation may be analyzed. Simultaneous recording of pulsed Doppler waves in an artery and a vein, respectively the aorta and the superior vena cava, has proven useful in the diagnosis of this arrhythmia. In this registration the atrial activity is rendered by the atrial reverse in the superior vena cava, and the ventricular activity by the aortic flow. This method is useful in determining the atrioventricular intervalthe correspondent of the PR interval on the electrocardio- 


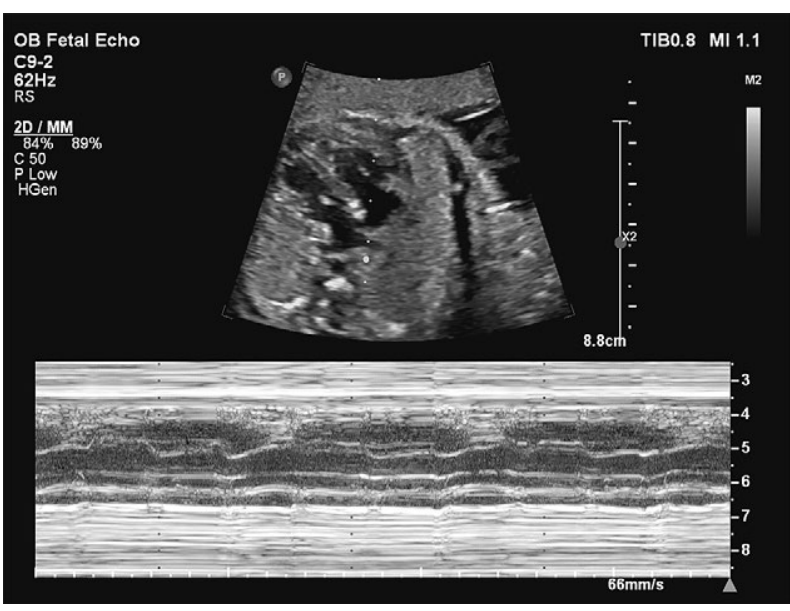

Fig 1. M mode recording: slow ventricular rate and complete dissociation between atrial and ventricular contractions.

gram, and it is of crucial importance to be monitored in pregnant women with a positive serology [3].

Given the very low frequency of this congenital pathology, the controversial data currently existing in the literature on treatment and evolution, we consider it important to share our experience on this topic, by presenting the cases from our clinical experience, respectively their immediate and long-term evolution.

\section{Material and methods}

In the period between 2013-2020, five fetuses were diagnosed and followed with CAVB in the Pediatric Cardiology Clinic from the Emergency Institute of Cardiovascular Diseases and Heart Transplant of Târgu-Mureș. In 4 of the cases mothers were asymptomatic in terms of autoimmune disease, but with positive serology with anti Ro/SSA antibodies, and in one case the mother was known to have Sjögren's syndrome. In each case, the

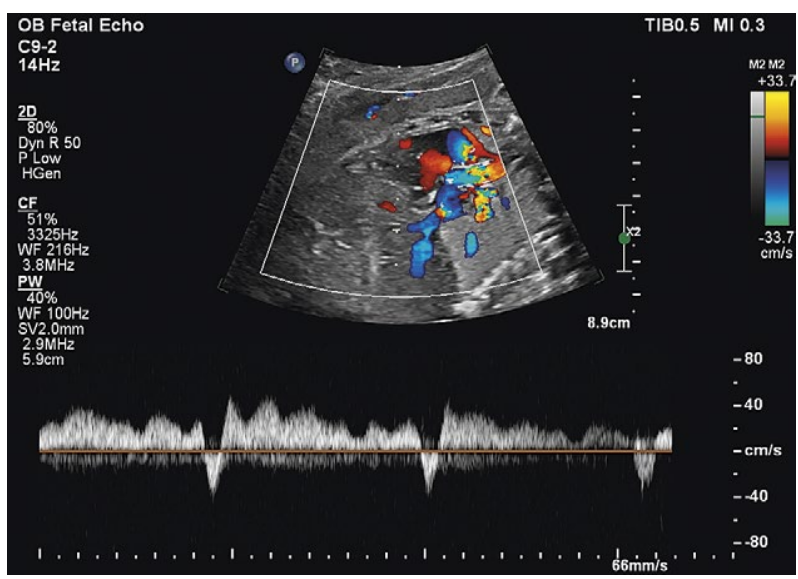

Fig 3. Doppler flow in the ductus venosus with atrial reversal

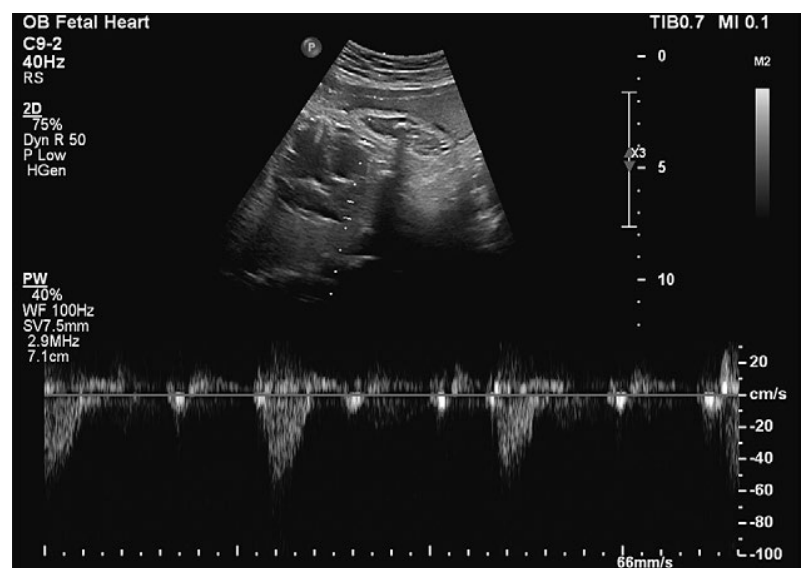

Fig 2. Pulsed Doppler recording in the ascending aorta and superior vena cava: dissociation between atrial and ventricular contraction

following echocardiographic diagnostic protocol was performed: establishing the anatomy of the fetal heart and excluding any structural abnormalities through a segmental sequential analysis; establishing the relationship between atrial and ventricular activity, using $\mathrm{M}$ mode (fig 1) and pulsed Doppler recorded in an artery and a vein, most commonly in the aorta and superior vena cava (fig 2); determination of the atrial and ventricular rates; hemodynamic evaluation of the fetus by calculating the fetal cardiovascular profile score (CVPS) $[4,5]$.

CVPS was determined taking into account each of the 5 echocardiographic elements: 1. hydrops (effusion -1 ; skin edema -2), 2. venous Doppler pulsation (atrial reversal in ductus venosus -1 fig3; umbilical vein atrial pulsation - 2), 3. umbilical artery Doppler flow (absent enddiastolic flow -1, reversed diastolic flow -2), 4. heart size (cardiothoracic ratio $0.35-50 \%-1$; cardiothoracic area $>$ $50 \%$-2 - fig 4), 5. cardiac function (RV / LV shortening

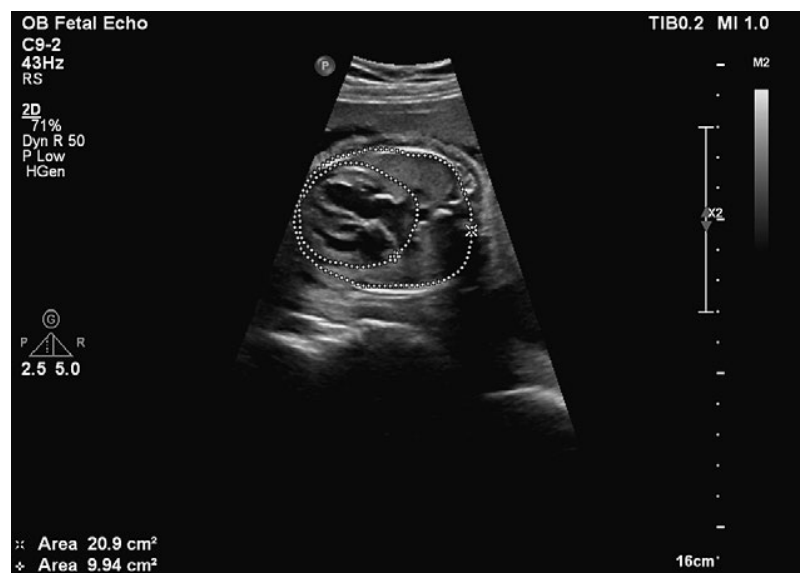

Fig 4. Cardiothoracic ratio 
fraction $<0.28$ or holosystolic tricuspid regurgitation -1 , mitral regurgitation or monophasic ventricular diastolic filling -2) [4].

To assist in the quantification of the ventricular function, in two of the cases we applied speckle tracking, by using a Qlab 13 software. Considering that the new speckle tracking analysis software allows the delimitation of the cardiac cycle without the need for a simultaneous electrocardiographic recording, the marking of the cardiac cycle being based upon the closure and aperture of the mitral valve (fig 5), we applied this method of assessing ventricular function in fetuses and newborn patients with atrioventricular block. The analysis is made in a four chamber view, recorded with a frame rate of at least $80 \mathrm{~Hz}$ (fig 6, fig 7)

\section{Results}

The clinical characteristics and the evolution of the cases are presented in Table I.

The therapeutic approach of the fetuses with complete atrioventricular block consisted of oral Dexamethasone, $4 \mathrm{mg}$ /day in a single dose for a period of 6 weeks. If an improvement was obtained, more precisely if the regression of the complete atrioventricular block to a second-degree atrioventricular block or even to periods of sinus rhythm was observed, the treatment was continued until the end of the pregnancy. If no enhancement was detected in atrioventricular conduction, dexamethasone therapy was discontinued. In the case of congenital AVB with low ventricular rate, we initiated betamimetic therapy (Salbutamol) for a period of time. No side effects of corticosteroid therapy were observed in any of the patients after birth.

Postnatal therapy involved the following: administration of Dopamine and Adrenaline- if the heart rate was extremely low and there were any signs of heart failure; followed by implantation of a pacemaker after a few days, with epicardial pacing wires placed on the left ventricle and device programming in VVI mode. Therapy and evolution of the cases are shown in table II.

Table I. Clinical characteristics and the evolution of the cases

\begin{tabular}{lllll}
\hline $\begin{array}{l}\text { Case } \\
\text { no. }\end{array}$ & $\begin{array}{l}\text { Gestational } \\
\text { age } \\
\text { (weeks) }\end{array}$ & $\begin{array}{l}\text { Heart } \\
\text { rate }\end{array}$ & $\begin{array}{l}\text { Fetal } \\
\text { cardiovascular } \\
\text { profile score }\end{array}$ & Hydrops \\
\hline 1 & 23 & $50 \mathrm{bpm}$ & 5 & Yes \\
2 & 24 & $68 \mathrm{bpm}$ & 9 & No \\
3 & 26 & $60 \mathrm{bpm}$ & 9 & No \\
4 & 24 & $50 \mathrm{bpm}$ & 7 & No \\
5 & 24 & $70 \mathrm{bpm}$ & 9 & No \\
\hline
\end{tabular}

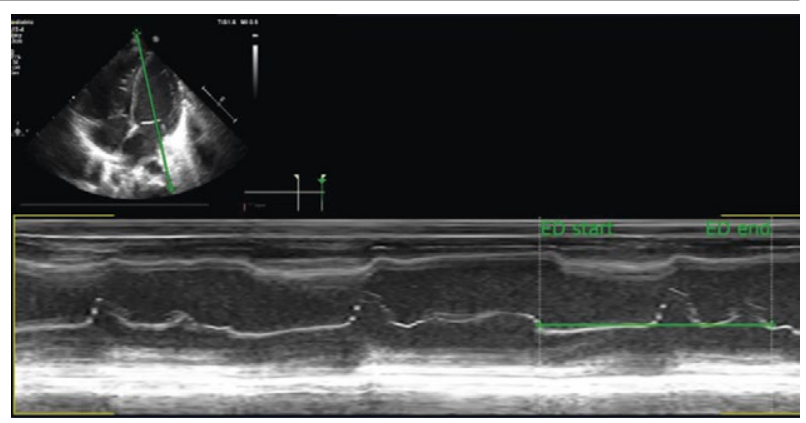

Fig 5. Delimitation of the cardiac cycle for speckle tracking analysis, using $\mathrm{M}$ mode

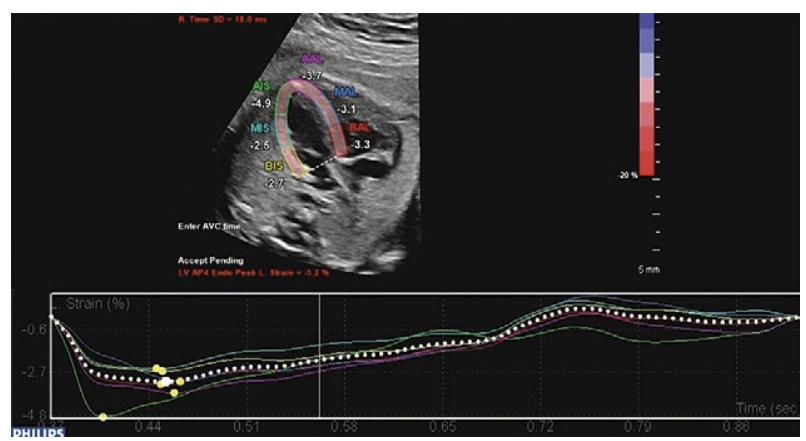

Fig 6. Four chamber view: longitudinal strain curves, in the fetal period

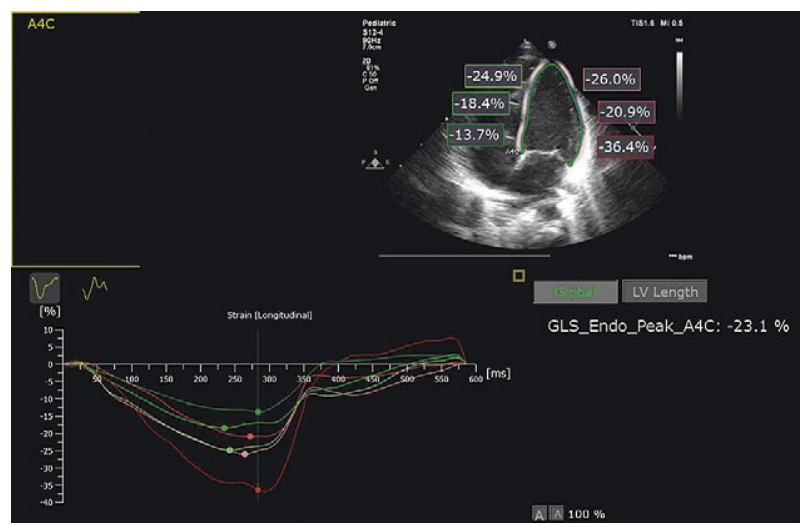

Fig 7. Four chamber view: longitudinal strain curves, in the neonatal period

\section{Discussion}

The first case of atrioventricular block was described in 1901 by Morqui. In 1972 the first studies were published, which reported the incidence of this pathology to be about 1 in 22,000 live births. [6,7].

Regarding the prevalence of this bradyarrhythmia among newborns from mothers with Ro / SSA antibodies, the first prospective study was performed by Brucato, who followed 100 seropositive women, respectively 118 pregnancies. From the total number of pregnancies fol- 
Table II. Therapy and evolution of the cases

\begin{tabular}{|c|c|c|c|c|}
\hline Case no. & Intrauterine treatment & Intrauterine evolution & Postnatal treatment & After birth evolution \\
\hline 1 & Dexamethasone & Intrauterine death & - & - \\
\hline 2 & Dexamethasone for 15 weeks & $\begin{array}{l}\text { Partial recovery of } \\
\text { atrioventricular conduction }\end{array}$ & $\begin{array}{l}\text { Monitoring and pacemaker } \\
\text { implant at } 14 \text { days of age }\end{array}$ & Favourable \\
\hline 3 & Dexamethasone for 6 weeks & Unchanged & $\begin{array}{l}\text { Pacemaker implant at } 7 \text { days } \\
\text { of age }\end{array}$ & Favourable \\
\hline 4 & $\begin{array}{l}\text { Dexamethasone and Salbutamol } \\
\text { for } 6 \text { weeks } \\
\text { Hydroxychloroquine }\end{array}$ & Slow unfavourable & $\begin{array}{l}\text { Dopamine Pacemaker implant } \\
\text { at } 6 \text { days of age }\end{array}$ & Favourable \\
\hline 5 & Dexamethasone for 6 weeks & Unchanged & No & Favourable \\
\hline
\end{tabular}

lowed, 2 fetuses were diagnosed with congenital complete heart block (CCHB), thus concluding that the prevalence of this pathology was low, up to $1.8-2 \%$ [8]. According to a study published by Tsubai, the risk of atrioventricular block is higher in the presence of an increased titre of maternal antibodies [9].

The occurrence of this major conduction disorder is secondary to cardiac injury resulting from the transplacental passage of maternal IgG antibodies. Although the number of seropositive mothers is high, the frequency of complete atrioventricular block in the fetus is still low $[1,10]$.

A study published by Askanase et al in 2002 highlights the progressive nature of the rhythm disorders in fetuses of seropositive mothers. Maternal antibodies cross the placenta and produce inflammatory changes in the fetal myocardium, followed by changes in myocytes and fibrosis. In certain situations, the process continues even after the disappearance of maternal antibodies, as evidenced by the progressive postnatal character: evolving from a minor disorder, prolonged PR interval highlighted at birth, to the appearance of complete atrioventricular block during childhood [10].

Prenatal diagnosis of fetal arrhythmias is difficult and involves an indirect assessment of fetal heart activity. It is mandatory that the diagnostic protocol includes both M-mode and pulsed Doppler examination, due to the fact that a second-degree atrioventricular block may be difficult to differentiate from blocked atrial extrasystoles [3].

Intrauterine fetal demise was estimated at $6 \%$ of fetuses with this pathology. After birth, death is rare and often due to cardiomyopathy associated with atrioventricular block [11].

Regarding the therapy of this pathology, the data published in literature are limited and somewhat controversial. The most common treatment options include steroids and beta-stimulation, immunoglobulins and hydroxychloroquine. Administration of dexamethasone may improve pleuro-pericardial effusions and intra- uterine evolution [12]. Studies published so far in the literature do not clarify the role of steroid therapy in first-degree atrioventricular block. It is difficult to prove the progression of first-degree atrioventricular block (diagnosed echocardiographically in M-mode or by pulsed Doppler as a prolongation of the atrioventricular interval) to second-degree atrioventricular block and even more difficult to prove the role of corticosteroid therapy in this setting [13-15].

Most fetuses diagnosed with second-degree atrioventricular block have developed complete atrioventricular block $[10,16]$ and there are extremely few cases in which an improvement in atrioventricular conduction has been achieved, once CAVB has been diagnosed. Data published in the literature regarding corticosteroid therapy in the setting of second-degree atrioventricular block are also controversial. Some studies report the recovery of atrioventricular conduction after steroid therapy in the context of a second-degree block $[12,17,18]$.

In a study consisting of 214 cases with high-degree congenital heart block, published by Levesque et al [19], the role of corticosteroids in the recovery of atrioventricular conduction was not proven. In the aforementioned study a case of complete atrioventricular block that recovered atrioventricular conduction without treatment was also reported [19]. Of the 5 cases we followed, case 2 had a similar evolution, with the recovery of atrioventricular conduction. At the age of 24 weeks, the fetus had a complete atrioventricular block, but at gestational age of 32 weeks, a partial resumption of atrioventricular conduction was observed. At birth, Holter ECG monitoring revealed long periods of sinus rhythm alternating with short periods of third-degree atrioventricular block, with supra-Hisian escape rhythm. Although the newborn was asymptomatic, at 4 weeks of age, pacemaker implantation was performed. Upon follow-up, currently at the age of 6 , the patient has long episodes of sinus rhythm with short periods of pacemaker rhythm. This evolution with the regression of a complete atrioventricular block 
is unusual and extremely rarely described in the literature [20]. To the best of our knowledge, this is the second case reported in the literature, in which an important recovery of atrioventricular conduction was obtained.

Cuneo et al draws attention to the existence of a transitional period until the appearance of complete atrioventricular block and emphasizes on the importance of intrauterine diagnostics and early initiation of therapy [21].

The last of the presented cases showed a favourable evolution throughout the pregnancy, with a heart rate of $70 \mathrm{bpm}$, without hemodynamic changes. During the neonatal period, heart rates of $80 \mathrm{bpm}$ were maintained, displaying an aspect of complete atrioventricular block, with a supra-Hisian escape rhythm. Furthermore, the assessment of ventricular function through speckle tracking, performed postnatal, showed very good longitudinal strain parameter values, but with a slight asynchrony in the contraction of the ventricular segments.

Despite numerous controversial data regarding corticosteroid therapy, it should not be discouraged [18]. Moreover, Sonnesson et al recently published a prospective study and concluded that steroid therapy has a positive effect on both the intrauterine and postnatal evolution, on the rate of the escape rhythm and could help increase the age at which the pacemaker is implanted [22].

Given the controversy surrounding intrauterine therapy, the monitoring the fetal heart with complete AVB is of extreme importance. Risk factors for an unfavourable evolution are: ventricular rate below $55 \mathrm{bpm}$, the appearance of endomyocardial fibroelastosis and low shortening fraction. With the help of the speckle tracking method, the ventricular function can be evaluated more faithfully, as well as any early evidence of a possible ventricular dysfunction. In case 4, although the heart rate and the fetal cardiovascular profile score (CVPS) were low, having a severely altered ejection fraction, global and segmental longitudinal strain and strain rates showed values close to normal, indicating a favourable prognosis [23]. Furthermore, in the last case, the good ventricular function, with very good longitudinal global and segmental strain values, helped us make the decision to delay the implantation of the pacemaker. It is known that complete atrioventricular block leads in time to the appearance of dilated cardiomyopathy, thus evaluation of ventricular function through speckle tracking can bring useful information to help therapeutic decision-making.

None of the 5 patients that were born presented any side effects from the administered corticosteroid therapy, and their evolution following the pacemaker implantation was favourable, with the normalization of echocardiographic parameters of the left ventricle; proving that the placement of epicardial electrodes on the left ventricle in pediatric patients with complete atrioventricular block is extremely important [24].

The limitations of this study are given primarily by the low number of cases with complete congenital atrioventricular block and the fact that the speckle tracking evaluation method has not yet entered into clinical practice, thus proving its importance in the functional evaluation of the fetal heart.

\section{Conclusions}

Because the prevalence of this pathology is very low, each clinical case must be analyzed in the light of current data available in the literature. The effectiveness of corticosteroid therapy is uncertain. Echocardiographic follow-up and completion of the evaluation protocol with longitudinal, global and segmental speckle tracking has an important role in the therapeutic decision.

\section{Conflict of interest: none}

\section{References}

1. Clancy RM, Kapur RP, Molad Y, Askanase AD, Buyon JP. Immunohistologic evidence supports apoptosis, IgG deposition, and novel macrophage/fibroblast crosstalk in the pathologic cascade leading to congenital heart block. Arthritis Rheum 2004;50:173-182.

2. Bordachar P, Zachary W, Ploux S, Labrousse L, Haissaguerre M, Thambo JB. Pathophysiology, clinical course, and management of congenital complete atrioventricular block. Heart Rhythm 2013;10:760-766.

3. Hunter LE, Simpson JM. Atrioventricular block during fetal life. J Saudi Heart Assoc. 2015;27:164-178.

4. Falkensammer CB, Paul J, Huhta JC. Fetal congestive heart failure: correlation of Tei-index and Cardiovascular-score. J Perinat Med 2001;29:390-398.

5. Huhta JC. Guidelines for the evaluation of heart failure in the fetus with or without hydrops. Pediatr Cardiol 2004;25:274-286.

6. Michaëlsson M, Engle MA. Congenital complete heart block: an international study of the natural history. Cardiovasc Clin 1972;4:85-101.

7. Kertesz NJ, Fenrich AL, Friedman RA. Congenital complete atrioventricular block. Tex Heart Inst J. 1997;24(4):301307.

8. Brucato A, Frassi M, Franceschini F, et al. Risk of congenital complete heart block in newborns of mothers with anti-Ro/SSA antibodies detected by counter immunoelectrophoresis: a prospective study of 100 women. Arthritis Rheum 2001;44:1832-1835.

9. Tsuboi H, Sumida T, Noma H, et al. Maternal predictive factors for fetal congenital heart block in pregnant mothers positive for anti-SS-A antibodies. Mod Rheumatol 2016;26:569-575. 
10. Askanase AD, Friedman DM, Copel J, et al. Spectrum and progression of conduction abnormalities in infants born to mothers with anti-SSA/Ro-SSB/La antibodies. Lupus 2002; 11:145-151.

11. Clowse MEB, Eudy AM, Kiernan E, et al. The prevention, screening and treatment of congenital heart block from neonatal lupus: a survey of provider practices. Rheumatology (Oxford) 2018;57(suppl_5):v9-v17.

12. Jaeggi ET, Fouron JC, Silverman ED, Ryan G, Smallhorn J, Hornberger LK. Transplacental fetal treatment improves the outcome of prenatally diagnosed complete atrioventricular block without structural heart disease. Circulation 2004;110:1542-1548.

13. Friedman DM, Kim MY, Copel JA, et al; PRIDE Investigators. Utility of cardiac monitoring in fetuses at risk for congenital heart block: the PR Interval and Dexamethasone Evaluation (PRIDE) prospective study, Circulation 2008; 117:485-493.

14. Jaeggi ET, Fouron JC, Silverman ED, Ryan G, Smallhorn J, Hornberger LK. Transplacental fetal treatment improves the outcome of prenatally diagnosed complete atrioventricular block without structural heart disease. Circulation 2004;110:1542-1548.

15. Rein AJ, Mevorach D, Perles Z, et al. Early diagnosis and treatment of atrioventricular block in the fetus exposed to maternal anti-SSA/Ro-SSB/La antibodies: a prospective, observational, fetal kinetocardiogram-based study. Circulation 2009;119:1867-1872.

16. Buyon JP, Hiebert R, Copel J, et al. Autoimmune-associated congenital heart block: demographics, mortality, morbidity and recurrence rates obtained from a national neonatal lupus registry. J Am Coll Cardiol 1998;31:1658-1666.

17. Saleeb S, Copel J, Friedman D, Buyon JP. Comparison of treatment with fluorinated glucocorticoids to the natural history of autoantibody-associated congenital heart block: retrospective review of the research registry for neonatal lupus. Arthritis Rheum 1999;42:2335-2345.

18. Ciardulli A, D’Antonio F, Magro-Malosso ER, et al. Maternal steroid therapy for fetuses with second-degree immune-mediated congenital atrioventricular block: a systematic review and meta-analysis. Acta Obstet Gynecol Scand 2018;97:787-794.

19. Levesque K, Morel N, Maltret A, et al. Description of 214 cases of autoimmune congenital heart block: Results of the French neonatal lupus syndrome. Autoimmun Rev 2015;14:1154-1160.

20. Bordachar P, Zachary W, Ploux S, Labrousse L, Haissaguerre M, Thambo JB. Pathophysiology, clinical course, and management of congenital complete atrioventricular block. Heart Rhythm 2013;10:760-766.

21. Cuneo BF, Ambrose SE, Tworetzky W. Detection and successful treatment of emergent anti-SSA-mediated fetal atrioventricular block. Am J Obstet Gynecol 2016;215:527528.

22. Sonesson SE, Ambrosi A, Wahren-Herlenius M. Benefits of fetal echocardiographic surveillance in pregnancies at risk of congenital heart block: single-center study of 212 antiRo52-positive pregnancies. Ultrasound Obstet Gynecol 2019;54:87-95.

23. DeVore GR, Cuneo B, Klas B, Satou G, Sklansky M. Comprehensive Evaluation of Fetal Cardiac Ventricular Widths and Ratios Using a 24-Segment Speckle Tracking Technique. J Ultrasound Med 2019;38:1039-1047.

24. Tissot C, Aggoun Y, Rimensberger PC, et al. Left ventricular epicardial VVI pacing for a congenital complete heart block with severe myocardial dysfunction: shall epicardial pacing wires be positioned left? Int J Cardiol 2007; 116:e7-e9. 